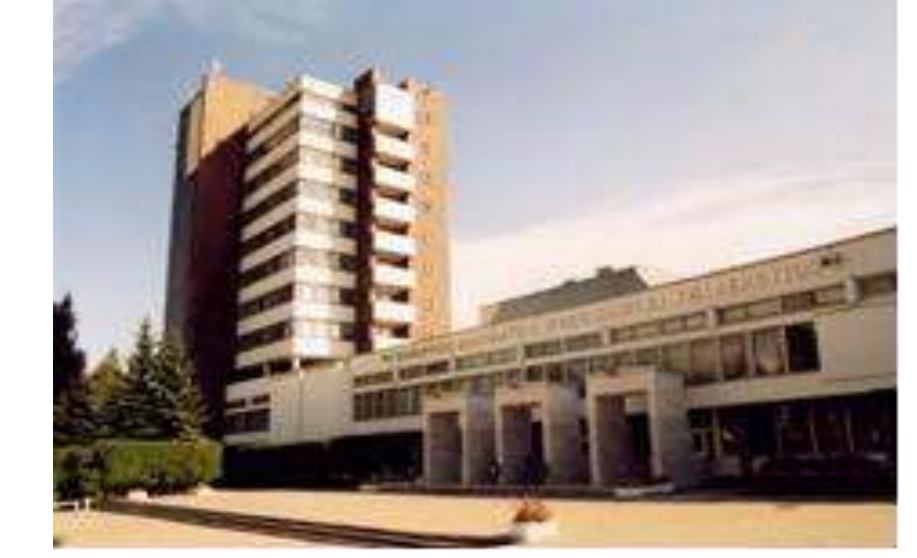

\title{
FEATURES OF HEART RHYTHM DISORDERS IN PATIENTS WITH TYPE 2 DIABETES
}

Shepelkevich, A. *(1); Dydyshka, Y.(1); Brutskaya - Stempkovskaya , E .(1)

${ }^{1}$ Department of Endocrinology, Belarusian State Medical University, Minsk, Belarus.

\section{BACKGROUND AND AIM:}

Currently, the diabetologists attention given to micro- and macrovascular complications of diabetes, while the first place among the causes of the death in the patients with type 2 diabetes (T2D) takes a cardiovascular pathology. Thus, the aim of the study was to reveal the features of heart rhythm disorders (HRD) in T2D patients as one of the factors determining the state of the cardiovascular system.

\section{SUBJECTS AND METHODS:}

- 66 T2D patients (34 women, 32 males) and 30 control subjects (15 women, 15 males) with a variety of HRD were examined. In the study carried out a detailed clinical examination of patients with medical records analysis. The patients with T2D and the control were comparable in sex, age and anthropometric data (Table1).

Table 1 - Clinical and laboratory characteristics of the examined patients with T2DM and control subjects, Me (LQ-UQ)

\begin{tabular}{|l|c|c|c|}
\hline Characteristic & T2DM & Controls & $\mathrm{p}$ \\
\hline Age (yrs) & $66.3(59.5-69.5)$ & $62.5(55.0-64.0)$ & $\mathrm{U}=58, \mathrm{p}=0,072$ \\
\hline Disease duration (yrs) & $7.0(4.5-9.0)$ & - & - \\
\hline Age of T2D onset (yrs) & $58.5(54.0-62.5)$ & - & - \\
\hline BMI (kg/m2) & $33.8(29.8-38.8)$ & $30.9(28.9-31.9)$ & $\mathrm{U}=308 ; \mathrm{p}=0,085$ \\
\hline HвA1c, \% & $7.6(5.9-12.1)$ & $5.3(4.4-5.6)$ & $\mathrm{U}=10, \mathrm{p}=0.003$ \\
\hline Total protein, g/l & $73.8(69.8-77.8)$ & $77.3(73.1-79.8)$ & $\mathrm{U}=67, \mathrm{p}=0,160$ \\
\hline $\begin{array}{l}\text { Glomerular Filtration Rate } \\
(\text { CKD-EPI), ml/min/1.73м }\end{array}$ & $59.6(46.1-64.6)$ & $64.8(60.1-68.9)$ & $\mathrm{U}=61, \mathrm{p}=0.091$ \\
\hline
\end{tabular}

The research involved:

$\checkmark$ Anthropometry of patients

$\checkmark$ General clinic examination

$\checkmark$ In both groups, the median BMI was more than $30 \mathrm{~kg} / \mathrm{m}^{2}$, that indicates the obesity presence.

$\checkmark$ All patients had arterial hypertension $(\mathrm{AH})$ and chronic heart failure (CHF).

$\checkmark$ In addition, the study included Ambulatory blood pressure monitoring, Electrocardiography, Echocardiography, Holter monitoring in all patients.

\section{RESULTS:}

$\checkmark \quad$ All patients received antihypertensive therapy, mostly combined, allowing reaching target blood pressure (BP) in T2D patients and the control group: $130 / 80$ vs $140 / 90$ ( $p>0.05) \quad \mathrm{mm} \mathrm{Hg}$ respectively (Table2).

$\checkmark \quad$ Established higher levels of total cholesterol and the triglycerides in patients with T2D without authentic significance of the differences (Table2).

$\checkmark \quad$ According to the results of Holter monitoring supraventricular DHR in the form of paroxysmal atrial fibrillation (AF) were recorded in 13 (19.7\%) patients with T2D and in $6(20.0 \%)$ controls $(X 2=0.37$, $\mathrm{p}=0.541$ ). Permanent atrial fibrillation (PAF) was found in 34 (51.5\%) T2D patients and in 14 (46.7\%) of the control group ( $\left(x^{2}=0.19, p=0.650\right)$.

$\checkmark \quad$ In general, supraventricular DHR were comparable in the patients with T2D and the control subjects: 46 $(69.7 \%)$ vs $24(80.0 \%)$ patients, $(x 2=1.11, p=0.292)$. The study of HRD as ventricular extrasystole often recorded in T2D patients compared to the control group - $20(30.3 \%)$ vs $3(10.0 \%)$ patients $(x 2=0.09$; $\mathrm{p}=0.767)$.

\section{CONCLUSIONS:}

In $30.3 \%$ of the patients with T2D recorded severe heart rhythm disorders in the form of supraventricular arrhythmias.

Significant prevalence of Moderate and Severe grade of aortic regurgitation in T2D patients was revealed that can be the cause of hypertrophic left ventricular remodeling and a poor prognostic factor for the development of HRD in this category of patients.

\section{According to the results of echocardiography} in the patients with T2D significantly more compared to the control dominated the heavier aortic regurgitation (Moderate and Severe grade) $(x 2=18.13 ; \quad p<0.001)$, but there were no significant differences in mitral $(x 2=3.03$, $\mathrm{p}=0.211)$ and tricuspid ( $\mathrm{x} 2=1.92, \quad \mathrm{p}=0,388)$ regurgitation.

According to the historical data in 16 (24.2\%) patients with T2D established acute disease - 13 $(19.7 \%)$ people with acute cerebrovascular accident and $3(4.5 \%)$ with myocardial infarction. In the control group $3(10.0 \%)$ suffered a stroke $(F=0.027, \quad p=0,166)$. On the subsequent hospitalization the three persons from the group with T2D died. 